SECTION 31. Economic research, Finance, innovation.

\author{
Naumov Anatoly Aleksandrovich \\ candidate of technical Sciences, Associate Professor, \\ Center of Applied Mathematical Research, Novosibirsk, Russia \\ E-mail: A_A_Naumov@mail.ru
}

\title{
THE MODELS OF CO-MANAGEMENT PROJECTS
}

In the work of the peculiarities of construction of models of comanagement projects.

Key words: management, project, business.

\section{К МОДЕЛЯМ СОВМЕСТНОГО УПРАВЛЕНИЯ ПРОЕКТАМИ}

В работе исследованы особенности построения моделей совместного управления проектами.

Ключевые слова: управление, проект, бизнес.

В частности, такие модели могут быть применимы при анализе интеграционных процессов (см. [1], [2]). Показаны недостатки некоторых моделей, их слабые места, которые приводят к выводу, что применительно к реальным интеграционным проектам использование некоторых предлагаемых в литературе моделей представляется затруднительным.

Постановка задачи. В работе [3] рассмотрены подходы к анализу эффективности проектов, реализуемых совместно. Такие проекты предлагается представить в виде структуры из пяти последовательных проектов, отвечающих за работу академических НИИ, прикладных НИИ, ВУЗов, малых инновационных предприятий и крупных промышленных организаций. Заметим, что, во-первых, не все эти проекты обязательно должны присутствовать в структуре и, во-вторых, в общем случае проекты могут выполняться одновременно (с перекрытием во времени) и с гибкими механизмами взаимодействия между ними (взаимным финансированием, с использованием трансфертных цен, взаимодействием с внешними бизнеспроцессами и проектами и т.д.).

В [3] для оценивания эффективности проекта из пяти подпроектов (этапов) предлагается использовать метод чистого приведенного дохода $(N P V)$, а для этого сначала находятся приведенные инвестиции $\left(P_{i}^{-}(t=\right.$ $0), i=1,2, \ldots, 5)$, а затем - приведенные доходы $\left(P_{1}^{+}(t=0), i=1,2, \ldots 5\right)$ для каждого из этапов. Так, например, для нахождения приведенных инвестиций для первого этапа используется формула вида:

$$
P_{1}^{-}(t=0)=x_{1} \cdot \frac{1-(1+i)^{-n_{1}}}{i}=\sum_{l=1}^{n_{1}} x_{1} \cdot(1+i)^{-l}=x_{1} \cdot \sum_{l=1}^{n_{1}}(1+i)^{-l},
$$


в ней $P_{1}^{-}(t=0)$ - дисконтированная на момент времени $t=0$ текущая стоимость потока платежей инвестиций на первом этапе проекта. Здесь $n_{1}$ - длительность первого этапа в годах; $x_{1}$ - величина годового платежа; $i$ ставка дисконтирования. На самом деле (фактически) здесь использована формула для нахождения значения размера кредита $P_{1}^{-}(t=0)$, выданного на $n_{1}$ лет, под процентную ставку $i$ и с равными ежегодными выплатами в размере $x_{1}$. Если речь идет действительно о погашении кредита, выданному первому проекту (под реализацию первого этапа), тогда необходимо отделить поток погашения кредита (в размере $x_{1}$ ежегодно) от потока вложений в проект, который может отличаться (по годам первого периода) от значений $x_{1}$. Пусть этот поток будет иметь вид (для первого этапа): $X_{1}=\left(x_{11}, x_{12}, x_{13}, \ldots, x_{1 n_{1}}\right)$. Тогда приведенный в точку $t=0$ поток инвестиций будет равен:

$$
P_{1, I}^{-}(t=0)=\sum_{l=1}^{n_{1}} x_{1 l} \cdot(1+r)^{-l} .
$$

Здесь $r$ - ставка дисконтирования отличная от ставки $i$, под которую выдан кредит. Заметим, что корректнее было бы использовать обозначения для ставок в виде $i_{1}$ и $r_{1}$, подчеркивая тем самым, что они различны на различных этапах проекта. Аналогично вычисляются выплаты по кредитам и приведенные в точку $t=0$ инвестиционные потоки для других этапов. Так, например, для второго этапа соответствующие формулы будут иметь вид:

$$
\begin{gathered}
P_{2}^{-}(t=0)=x_{2} \cdot \frac{1-\left(1+i_{2}\right)^{-n_{2}}}{i\left(1+i_{2}\right)^{n_{1}}}, \\
P_{2, I}^{-}(t=0)=\sum_{i=1}^{n_{2}} x_{2 i} \cdot\left(1+r_{2}\right)^{-i} .
\end{gathered}
$$

Здесь $i_{2}$ - ставка по кредиту второго этапа и $r_{2}-$ ставка дисконтирования. Отметим, что если потоки разных этапов не зависят друг от друга (не влияют друг на друга), то, во-первых, нет необходимости приводить поток инвестиций к одному и тому же моменту времени $(t=0)$ на каждом из этапов и, во-вторых, эти показатели не дают оснований для оценивания эффективности всей интегрированной структуры, а лишь для отдельных независимых частей (этапов) проекта. К сожалению, следует заметить, что часто в работах по анализу интегрированных процессов на эффективность в описательной части рассматриваются особенности интеграционных процессов, их основные характеристики, их отличие от других процессов (в том числе и от инвестиционных процессов), а когда речь заходит об оценивании их эффективности, расчетные схемы обычно заимствуются без адаптации и доработок из множества схем расчета эффективности для обычных инвестиционных проектов. На наш взгляд, конечно, расчетные схемы и сам подход к оцениванию эффективности интеграционных процессов должны отражать суть интеграции. Например, к таким особенностям относятся: 1) перекрытие во времени областей функционирования отдельных проектов (частных бизнес-процессов) в рамках интеграционного процесса; 2) выделение и передача части своих 
бизнес-процессов частными процессами интеграционным процессам; 3) эффективность частных процессов является производной и определяется эффективностью всего интеграционного процесса и т.д. Последнее, в частности, означает, что сначала должна оцениваться эффективность интеграционного процесса в целом, а уже затем - эффективности образующих его частных проектов (бизнес-процессов). Отметим еще одну особенность расчетных схем для анализа интеграционных (и не только) процессов на эффективность.

Перейдем к анализу доходных частей проекта. В [3] предлагается на первом этапе дисконтированный поток доходов рассчитать по формуле:

$$
\begin{aligned}
& P_{1}^{+}(t=0)=x_{1} \cdot \frac{1-(1+i)^{-n_{1}}}{i} \cdot k_{1} \cdot \frac{1}{(1+i)^{n_{1}}}= \\
& \quad=P_{1}^{-}(t=0) \cdot k_{1} \cdot \frac{1}{(1+i)^{n_{1}}}, \quad k_{1} \geq 1,
\end{aligned}
$$

где $P_{1}^{+}(t=0)$ - приведенный на момент времени $t=0$ поток доходов первого этапа (кстати, поскольку $P_{1}^{-}(t=0)$ привязан к началу первого этапа, то диконтировать это значение не нужно); $k_{1}-$ коэффициент доходности инвестиций. Аналогично расчитываются приведенные доходные части для остальных этапов. Замечание, касающееся операции дисконтирования для формулы (1), остается в силе и здесь. После того, как будут найдены все составляющие вида $P_{i}^{-}(t=0), i=1,2, \ldots, 5$, и $P_{i}^{+}(t=$ $0), i=1,2, \ldots, 5$, для оценивания эффективности интеграционного проекта (включающего пять этапов), в предлагается воспользоваться формулой для $N P V$ :

$$
N P V\left(x_{1}, x_{2}, x_{3}, x_{4}, x_{5}, y_{5}\right)=\sum_{i=1}^{5}\left(P_{i}^{+}(t=0)-P_{i}^{-}(t=0)\right)=\sum_{i=1}^{5} N P V_{i} \text {. (6) }
$$

Здесь $y_{5}$ - доход пятого этапа. Задача (6), естественно, разбивается на пять независимых подзадач (для каждого этапа в отдельности) построенная модель описывает не интеграционный процесс, а проект, разбитый на последовательность независимых этапов. Как следовало бы решать задачу оценивания эффективности интеграции в общем случае? Схема может быть такой (см., например, [2], [4]). На первом этапе (шаге) решения такой задачи выстраивается структура интеграционного процесса (проекта). Затем для полученной структуры интеграционного бизнеспроцесса оцениваются доход и доходность ( $N F V, I R R$ и др.), а затем находятся доходы и доходности частных бизнес-процессов (проектов).

\section{Литература}

1. Плещинский А.С., Титов В.В., Межов И.С. Механизмы вертикальных взаимодействий предприятий (вопросы методологии и моделирования). - Новосибирск: ИЭОПП СО РАН, 2005. — 336 с.

2. Наумов А.А., Клавсуц И.Л., Лямзин О.Л. Инновации. Теория, модели, методы управления. - Новосибирск: ОФСЕТ, 2010. - 415 с. 
3. Кириллов Ю.В., Досужева Е.Е. Экономико-математическая модель поддержки принятия решений по инвестированию в совместные инвестиционные проекты, Финансовая аналитика: Проблемы и решения, 2013, № 27, c. 33-39.

4. Список трудов [Электронный pecypc]. URL: https://sites.google.com/site/anatolynaumov2011/home/spisok-trudov-list-ofpapers (дата обращения: 25.08.2013). 